\title{
A Principal Component Analysis of Acoustic Emission Signals from a Landing Gear Component
}

\author{
R. Pullin ${ }^{1, a}$, M. J. Eaton ${ }^{1, b}$, J. J. Hensman ${ }^{2, c}$, K. M. Holford ${ }^{1, d}$, K. Worden ${ }^{2, e}$ \\ and S. L. Evans ${ }^{1, f}$ \\ ${ }^{1}$ Cardiff School of Engineering, Queens Buildings, The Parade, Newport Road, Cardiff, CF24 \\ 3AA, U.K. \\ 2 Dynamics Research Group, Department of Mechanical Engineering, University of Sheffield \\ Mappin Street, Sheffield S1 3JD, U.K. \\ apullinr@cardiff.ac.uk, ${ }^{b}$ eatonm@cardiff.ac.uk, ${ }^{c}$ mep04jh@sheffield.ac.uk, ${ }^{d}$ holford@cardiff.ac.uk, \\ e.worden@sheffield.ac.uk,
}

\section{Keywords: Acoustic Emission, Principal Component Analysis, Aerospace}

\begin{abstract}
This work forms part of a larger investigation into fracture detection using acoustic emission (AE) during landing gear airworthiness testing. It focuses on the use of principal component analysis (PCA) to differentiate between fracture signals and high levels of background noise. An artificial acoustic emission (AE) fracture source was developed and additionally five sources were used to generate differing AE signals. Signals were recorded from all six artificial sources in a real landing gear component subject to no load. Further to this, artificial fracture signals were recorded in the same component under airworthiness test load conditions. Principal component analysis (PCA) was used to automatically differentiate between AE signals from different source types. Furthermore, successful separation of artificial fracture signals from a very high level of background noise was achieved. The presence of a load was observed to affect the ultrasonic propagation of AE signals.
\end{abstract}

\section{Introduction}

Aerospace components such as landing gear are required to pass strict air worthiness tests before being allowed into in-flight service. Landing gear components are required to complete a fracture resilience test, during which they must endure several life cycles of loading, lasting up to five years, without developing any fractures. During the fracture resilience test, regular NDT inspections are performed to assess the integrity of the component throughout and account for up to $25 \%$ of the test time. The use of acoustic emission (AE) monitoring has been proposed as a method of reducing the need for periodic NDT inspection.

A landing gear fracture resilience test has numerous noise sources such as hydraulic noise, bearing noise, friction between components and movement of sliding tubes. In addition there are numerous propagation paths that can dramatically alter the signal with respect to distance. Therefore the ability to distinguish between sources is very important for the successful identification of fatigue crack signals.

This paper expands on previous work using Principal component analysis (PCA) of feature data [1-3]. PCA is a method used to simplify high order data sets to lower dimensions to allow a simple analysis. For further explanation of PCA, see Nabney [4]. In this work it is shown how PCA can be used to distinguish between different artificial sources in a landing gear component and additionally how noise and artificial fracture signals can be separated during a real fracture resilience test. 


\section{Experimental Procedure}

In order to assess the ability of the PCA technique to distinguish between signals from fracture sources and noise in a landing gear structure, an artificial fatigue fracture source was designed. This was necessary because landing gear components must not develop any fatigue fractures during an airworthiness test in order to be deemed airworthy and, as such, real fractures are a rare occurrence. During a previous investigation using compact tension (CT) specimens, signals from fracture sources were collected [5]. Using a new undamaged specimen, with an identical AE set-up, signals were pulsed using a Physical Acoustics wave-generator (Fig. 1) at the crack location and the response monitored. The output of the wave-generator was adjusted until the received signal closely matched the previously recorded fracture signal. Five further signals were created to assess the ability of the PCA technique to distinguish between different sources.

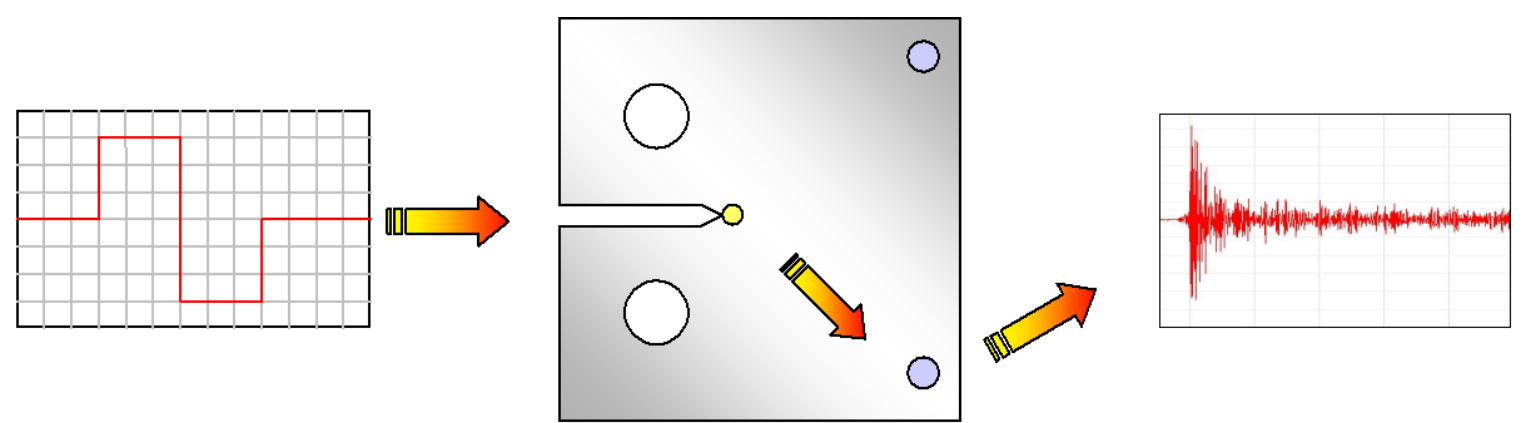

Fig. 1: Test set-up for the creation of the artificial source

Fig. 2 shows a comparison between a fracture signal and the designed artificial source, it can be seen that there is a close correlation between the two signals. Table 1 presents the feature data of the two signals, at first glance the feature data does not appear to be particularly closely matched, however when the maximum values of the individual features recorded during a fatigue test (i.e. durations in excess of $62,000 \mu \mathrm{s}$ and energy in excess of $180 \mathrm{E}+06 \mathrm{JJ}$ ) are considered the variation observed is not large. Rise time, amplitude and initiation frequency are all similar, whereas energy, duration and counts are slightly skewed by the longer ring down of the artificial signal, however adjustments made to correct this cause other signal features to alter. Table 2 provides details of the pulse used to produce the artificial fracture source, labelled Hann wave 1, plus the additional designed sources that are variations on Hann and sawtooth waves.
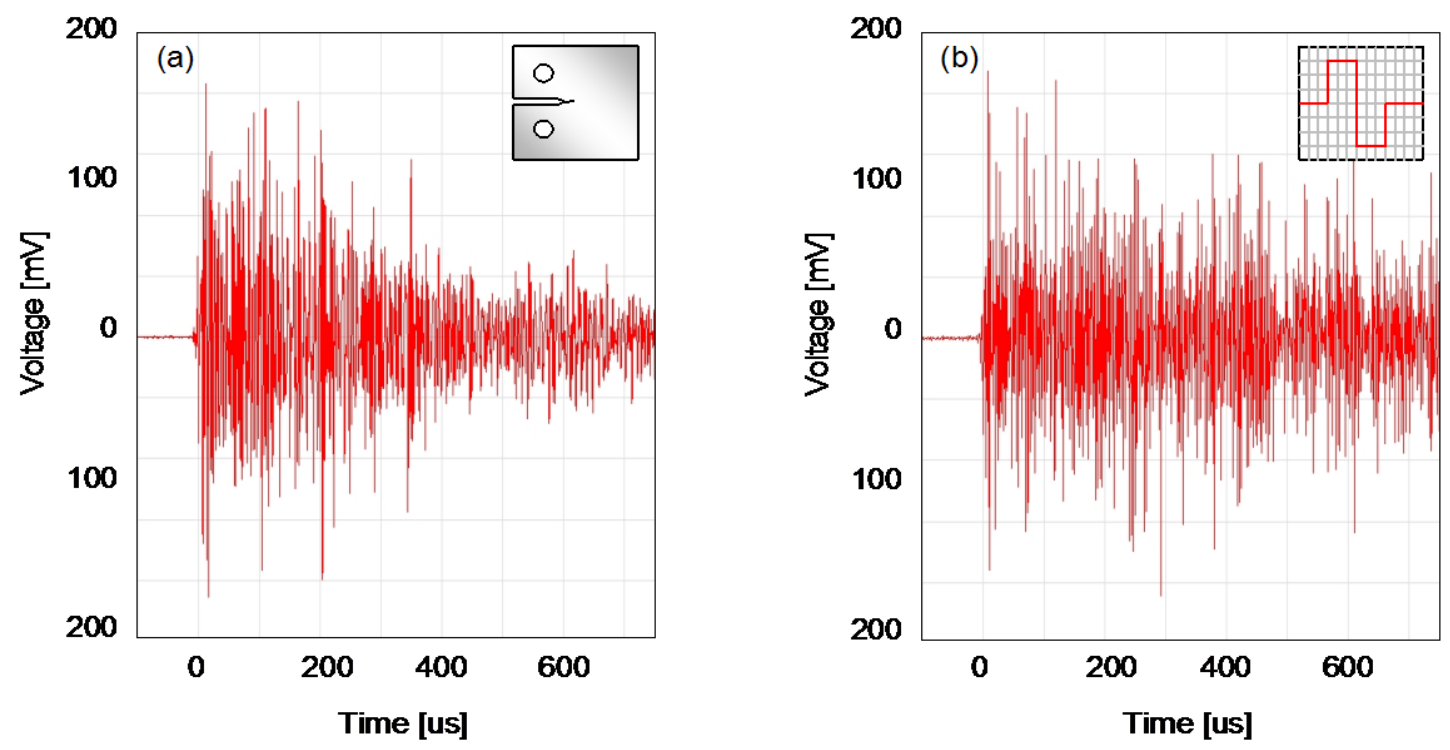

Fig. 2: Comparison of signals and feature data from a) fatigue fracture and b) artificial source 
Table 1: Comparison of artificial and real fracture signal feature data

\begin{tabular}{|c|c|c|c|c|c|c||}
\cline { 2 - 7 } \multicolumn{1}{c|}{} & $\begin{array}{c}\text { Rise Time } \\
{[\boldsymbol{\mu s}]}\end{array}$ & Counts & $\begin{array}{c}\text { Duration } \\
{[\boldsymbol{\mu s}]}\end{array}$ & $\begin{array}{c}\text { Amplitude } \\
{[\mathbf{d B}]}\end{array}$ & $\begin{array}{c}\text { Ini-Freq } \\
{[\mathrm{KHz}]}\end{array}$ & $\begin{array}{c}\text { Abs-Energy } \\
{[\mathbf{a J}]}\end{array}$ \\
\hline Crack & 18 & 132 & 940 & 67 & 278 & $17.250 \mathrm{E}+03$ \\
\hline Wavegen & 10 & 439 & 2907 & 68 & 400 & $45.140 \mathrm{E}+03$ \\
\hline
\end{tabular}

Table 2: Details of pulsed waves

\begin{tabular}{||l|c|c|c||}
\cline { 2 - 4 } \multicolumn{1}{c|}{} & $\begin{array}{c}\text { Amplitude } \\
{[\text { [V] }}\end{array}$ & Cycles & $\begin{array}{c}\text { Frequency } \\
{[\mathbf{k H z}]}\end{array}$ \\
\hline Hann 1 & 0.6 & 1 & 400 \\
\hline Hann 2 & 0.6 & 25 & 100 \\
\hline Hann 3 & 1.2 & 1 & 600 \\
\hline Sawtooth 1 & 0.6 & 1 & 400 \\
\hline Sawtooth 2 & 0.6 & 25 & 100 \\
\hline Sawtooth 3 & 1.2 & 1 & 600 \\
\hline
\end{tabular}

For the main investigation eight Physical Acoustics Ltd. (PAL) resonant sensors were attached to a landing gear component (Fig. 3). Ultrasound gel was used to couple the sensors to the surface whilst magnetic clamps were used to hold the sensors in position. A further pulsing source was added to the structure as shown in Fig. 2b. The six designed signals were then pulsed into the unloaded structure.

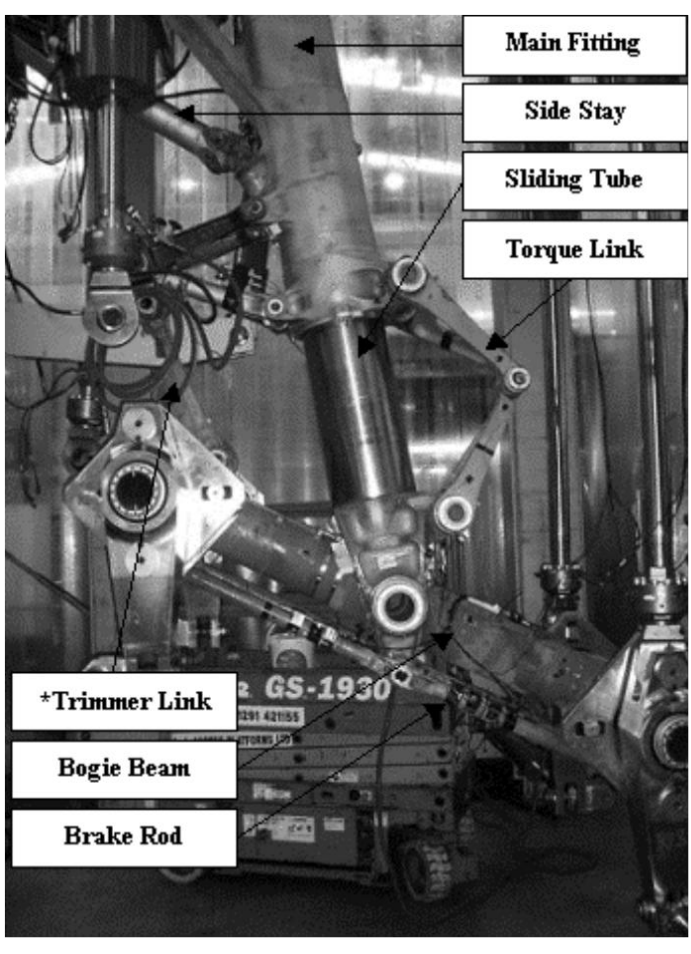

(a)

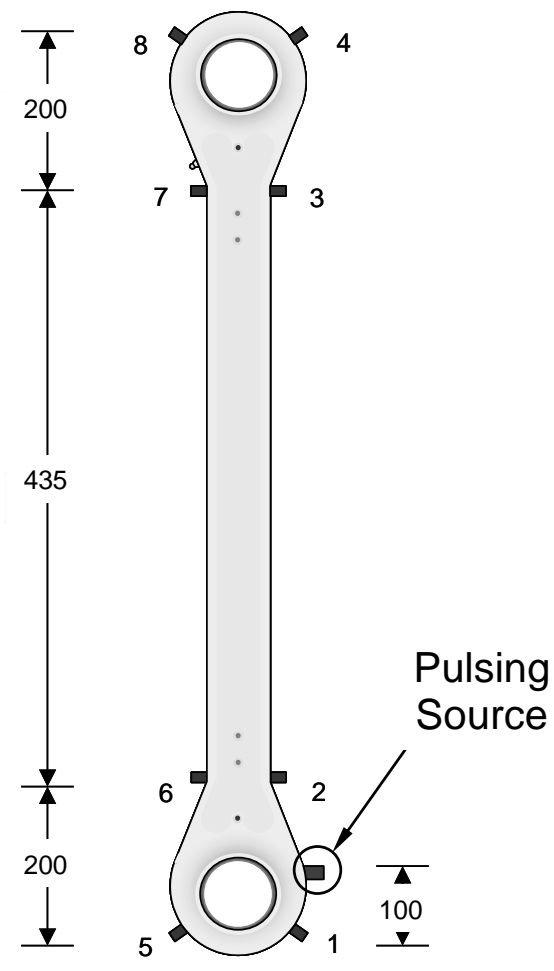

(b)

Fig. 3: Test Specimen (a) main landing gear component undergoing fracture resilience testing (b) instrumented trimmer link component

A further investigation was completed where the fracture signal was pulsed at $0.05 \mathrm{~Hz}$ for a period of 2,400s during full loading. Feature data was recorded for signals of $45 \mathrm{~dB}$ amplitude and above using a PAL DiSP system for all investigations. 


\section{Trimmer Link Investigation I}

Fig. 4 shows the response of sensor 2 in the trimmer link investigation (Fig. 3) to the six different pulsed signals (all plots have identical scales). It can be seen that that Hann wave 1 and 3 and sawtooth 1 and 3 are very close in appearance, this was un-intentional but provided a good examination of the PCA technique. A further investigation of the pulsed signal technique demonstrated that as the amplitude of the pulsed signal increased so did the sensor response, as expected, however as the frequency of the signal moved away from resonance of the pulser the amplitude decreased, essentially meaning there was relatively little difference in the two signals. The remaining two signals (Hann wave 2 and Sawtooth 2) however can be separated easily by visual inspection.

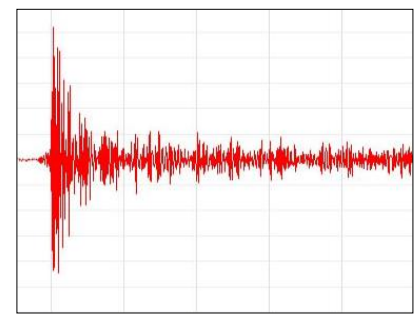

Han Wave 1

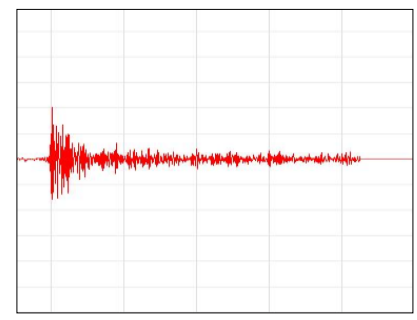

Sawtooth 1

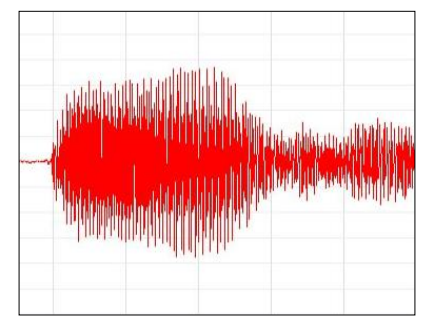

Han Wave 2

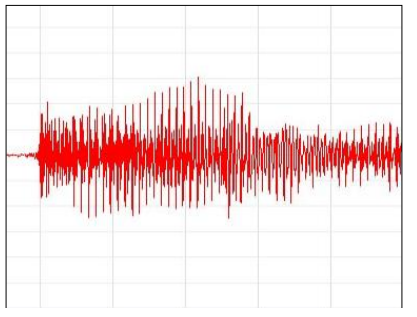

Sawtooth 2

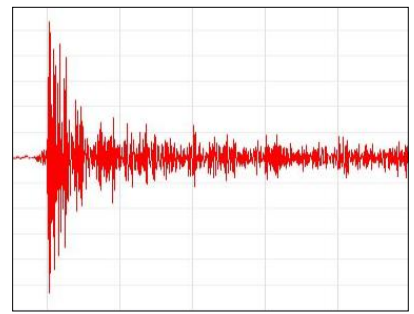

Han Wave 3

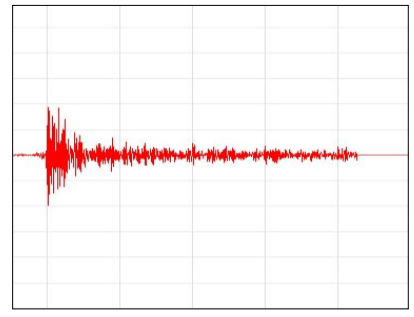

Sawtooth 3

Fig. 4: Response of sensor 2 to pulsed signals (all signals plotted on identical scales)

Fig. 5 shows the PCA of the six different artificial AE signals recorded by sensor 2, it can be seen that there is distinct separation between signals from sawtooth 2 and Hann wave 2, whilst signals from Hann wave 1 and 3 are grouped closely together and are separate from signals from sawtooth 1 and 3, which are overlapping. This demonstrates that the technique can successfully and automatically differentiate between signals that are distinctly different. It is not surprising that it was not possible to separate signals that are very similar, in fact the purpose of the technique is to identify signals that have fracture characteristics (i.e. distinctly different to that of signals from noise sources) which Hann wave 1 and 3 signals clearly exhibit.

The response of all eight sensors to the fracture like signal (Hann wave 1) is shown in Fig 6. An initial inspection shows that the majority of all fracture like signals remain distinctly separate to the non fracture sources (Fig. 5). The separation observed between signals recorded by channels 1-7 highlights the effect that differences in propagation path and sensor response can have on a recorded signal. The tight clustering of signals recorded at each individual channel demonstrates how the fracture like quality of a signal is maintained over reasonable propagation distances. In this example a simple filter based on the lower quadrant could be used to identify fracture signals. However this is unlikely to be achievable in a full landing gear investigation.

Sensor 8 is the exception in this example but it should be noted it has the most complicated propagation path from the source (Fig. 3) (i.e. not straight line propagation as it is to sensors 3 and 4) and the signal will be altered as it propagated through the structure. Furthermore in a practical 
investigation the first hit sensor, the closest to a source, would be used to perform the PCA eliminating the problem associated with differences in propagation path and sensor response.

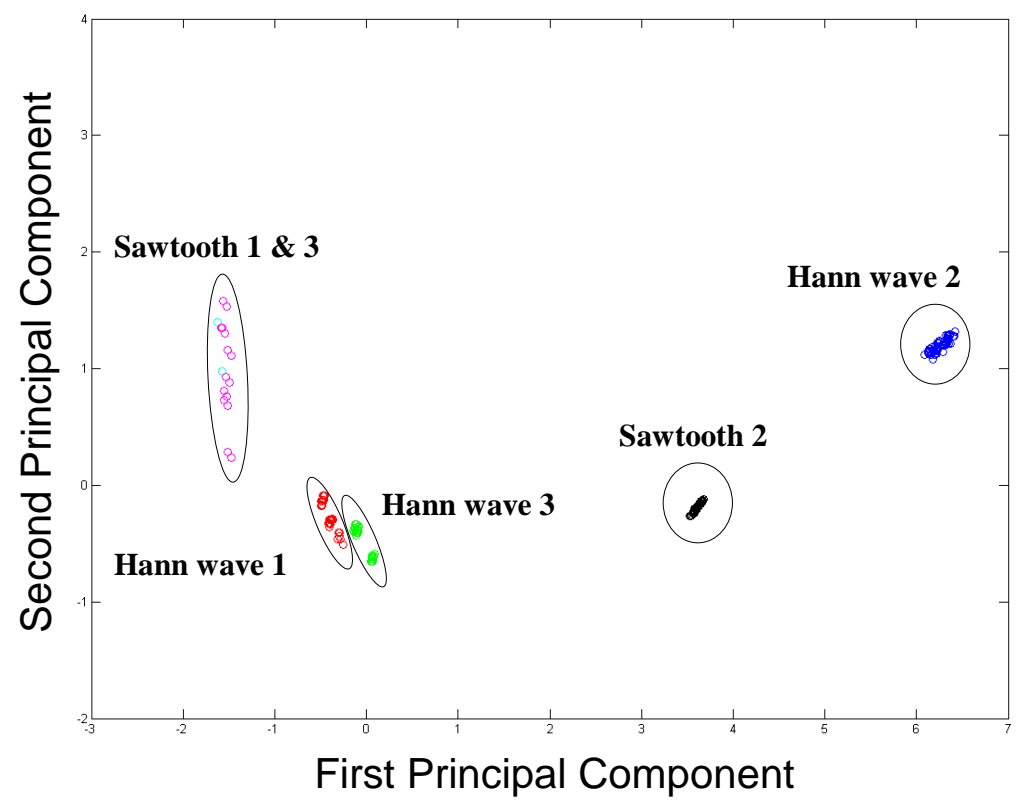

Fig. 5: Principal component analysis of 6 artificial waveforms recorded by sensor 2

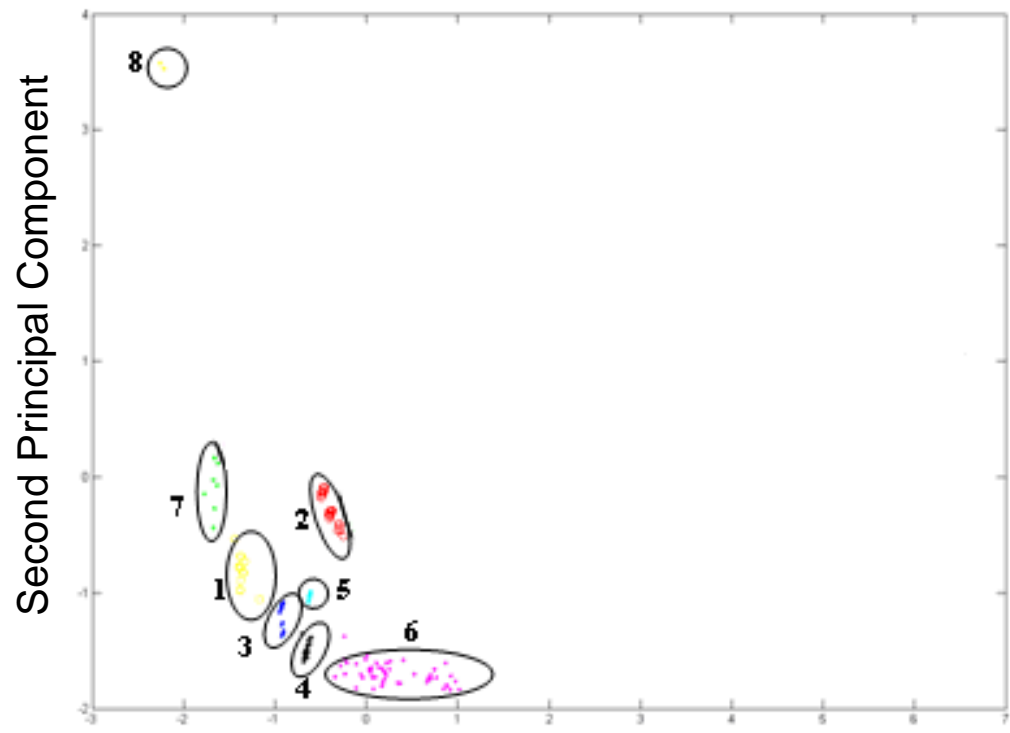

First Principal Component

Fig. 6: Principal component analysis of response at all sensors to Hann wave 1

\section{Trimmer Link Investigation II}

The time history of the detected signals recorded during the second trimmer link investigation is presented in Fig. 7, for a single flight cycle, in terms of the amplitude and the energy of the recorded signals. The plots demonstrate how a landing gear test is a high noise environment. With a pulse rate of $0.05 \mathrm{~Hz}$, there were approximately 10 artificial fracture sources for 2800 signals from noise, this is assumed to be a reasonable ratio of fracture signals per cycle. 

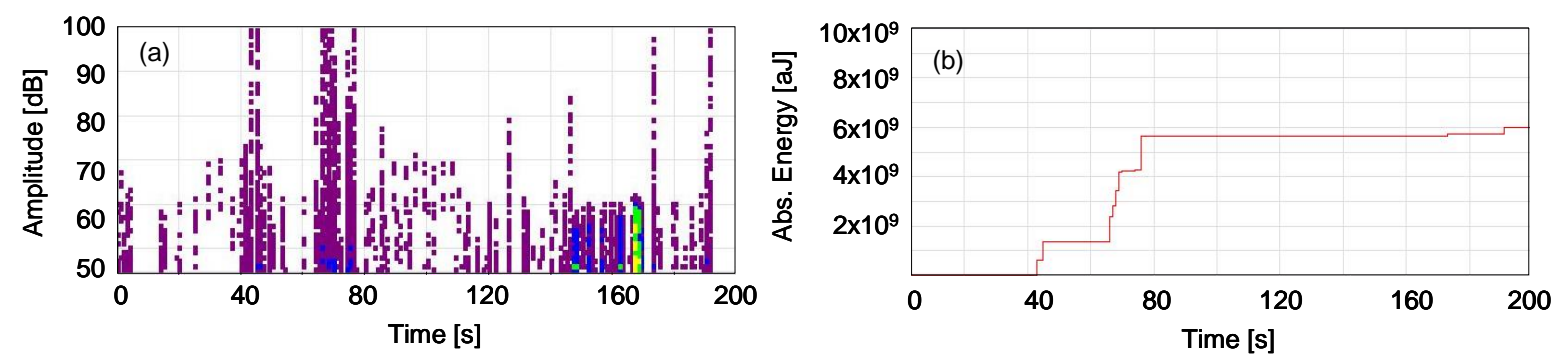

Fig. 7: History of detected signals for 1 cycle (a) amplitude of detected signals (b) Absolute energy of detected signals

A PCA of the signals located at the artificial fracture source and the remaining test signals (presumed to be rig noise) is presented in Fig. 8. The plot clearly shows a separation of the artificial source and landing gear noises. This demonstrates that the PCA technique is a suitable method for separating actual fatigue fracture signals from high levels of noise in airworthiness fracture resilience tests.

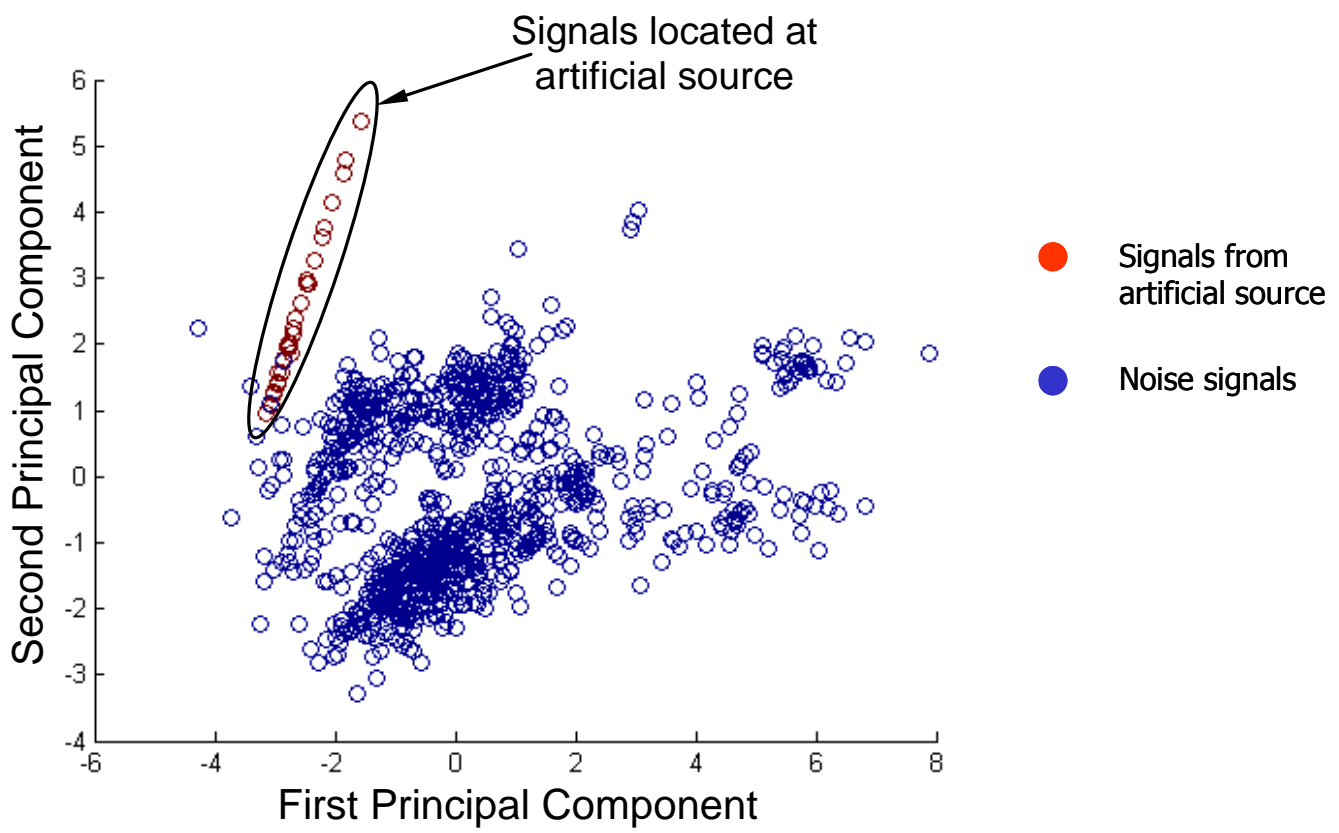

Fig. 8: PCA of artificial source and landing gear noise

A final PCA was performed to assess if there was any difference between the artificial source pulsed with and without the presence of loading, which is shown in Fig. 9. It can be seen there is a distinct difference in the detected signals. This is due to the effects of the strained signal as reported by Hensman [6]. However in a practical test it is expected that an AE source will be active at the same point in each load cycle (i.e. at the same strain), hence limiting this affect. It should also be noted that the variance observed in signals recorded at different strain levels is small relative to that of the noise signals seen in Fig. 8. 


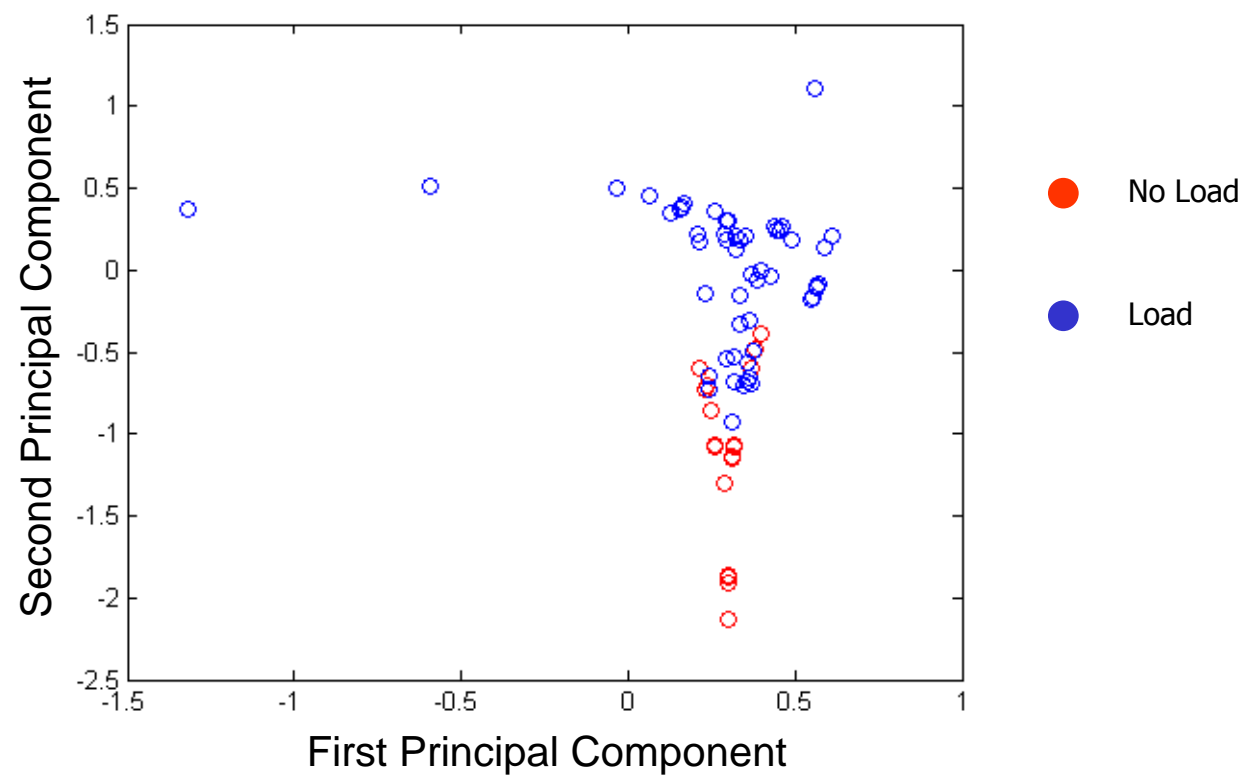

Fig. 9: PCA of artificial source pulsed with and without loading

\section{Conclusions}

An artificial fracture signal was successfully developed and used along with five other signals to investigate the PCA technique. The technique was used to automatically differentiate between distinctly different artificial signals in complex landing gear components, indicating the potential to discriminate between fracture and noise signals. Differences in propagation path and sensor response were shown to have an effect on the recorded signals however this can be overcome by using a single sensor to differentiate between signal types. A further investigation demonstrated how PCA can be used to separate artificial fracture signals from very high levels of test rig noise.

\section{Acknowledgements}

The authors would like to thank Tim Baker and David Bond of Messier-Dowty for their assistance. The financial support of EPSRC (Grant No. GR/T26757/01) and Messier-Dowty is also acknowledged.

\section{References}

[1] S. Rippengill, and K. Worden, and K. M. Holford and R. Pullin, Journal of Strain Vol. 39, No. 1, pp 31-41 (2003)

[2] G. Manson, and K. Worden, and K. M. Holford, and R.Pullin, Journal of Intelligent Material Systems and Structures Vol. 12, No. 8, pp, 529-536 (2001)

[3] R. Pullin, J. J. Hensman, K. M. Holford, K. Worden and S. L. Evans, Key Engineering Materials, Vols. 347, pp. 139-144, ISBN 0-87849-444-8 (2007)

[4] Nabney, I.T., Netlab: Algorithms for Pattern Recognitions, published by Springer (2004)

[5] M. Baxter, R. Pullin and K. M. Holford, 26th European Conference on Acoustic Emission Testing, Berlin, 15-17th September, ISBN 3-931-381-58-7, pp. 331-341 (2004)

[6] J. J. Hensman, C. V. Cristodaro, S. G. Pierce and K. Worden, Advanced Materials Research Vols. 13-14, pp 117-124 (2006) 